\title{
Segmentation of satellite images using Self-Organizing Maps
}

\author{
Mohamad Awad \\ National Council for Scientific Research-Center for Remote Sensing \\ Lebanon
}

\section{Introduction}

Remote sensing plays a key role in many domains devoted to observation of the Earth, such as land cover/use, agriculture monitoring, military battles, oceanography...etc. There are many types of acquisition systems which have different spatial, spectral, and temporal characteristics. Some of them are passive, such as Landsat, Spot, Ikonos, Quickbird, Orbview...etc. Others are active such as SAR (Space Airborne Radar). These systems have opened the field of applications since early 1970.

Image segmentation is the process of image division into regions with similar attributes (Pratt, 1991). It is an important step in image analysis chain with applications to pattern recognition, object detection, etc. Until recently, most of the segmentation methods and approaches are supervised such as Maximum A Posteriori (MAP) (Lopes et al., 1990) or Maximum Likelihood (ML) (Benediktson et al., 1990) with an average efficiency rate of about $85 \%$ (Perkins et al., 2000), (Zhang et al., 2003). In the supervised methods a priori knowledge is needed to get a successful segmentation process and sometime the required information may not be available. In addition, there are unsupervised methods which require many parameters and they are sensitive to noise such as Iterative Self-Organizing Map Data (ISODATA) (Tou \& Gonzalez, 1974), and SEM (Mason \& Pieczynski, 1993). In order to overcome the deficiencies found in many previously listed methods, Kohonen's Self-Organizing Maps (SOM) (Kohenen, 2001) is used to segment different satellites images. $\mathrm{SOM}$ is an unsupervised non-parametric Artificial Neural Network (ANN) method. The main characteristic of SOM is the ability to convert patterns of arbitrary dimensionality into the responses of two dimensional arrays of neurons. Another important characteristic of the $\mathrm{SOM}$ is that the feature map preserves neighborhood relations of the input pattern. Although the use of SOM in image segmentation is well reported in the literature, such as segmentation of printed fabric images (Xu \& lin, 2002), or in sonar images (Yao et al.,2000), their application in satellite image segmentation is not widely known. One can cite the work of (Aria et al., 2004) which was used in the segmentation of Indian Remote Sensing "IRS" satellite image. The cooperative segmentation approach between K-means and SOM (Zhou et al., 2007) is a recent work where the role of K-means is to segment the image in the coarser scale, and then SOM will re-segment the image in the fine scale. In This method K- 
means requires the pre-determination of cluster numbers and this kind of work can be taken care by SOM itself.

Another promising work is the cooperation between SOM and the Hybrid Genetic Algorithm (GA) (Awad et al., 2007). SOM provides many cluster centers and GA finds the optimal number of these centers. This cooperation was tested on different satellite images such as Landsat, Spot, Ikonos and the results were of high accuracy. However, the speed of image processing remains an important issue.

In this chapter, SOM based on a threshold technique and SOM cooperated with another method are tested on two different types of satellite images with different resolution. In the first method SOM is combined with the threshold based technique. In the cooperative segmentation method, SOM is combined with the Hybrid Dynamic Genetic Algorithm (HDGA) (Awad et al. 2009a) to segment the images. The goal is to check the efficiency of SOM with and without cooperation with another algorithm. In addition, the use of HDGA is to increase the speed of processing.

After this introduction, SOM complete details will be covered in the second section. The third section covers the combination of SOM and threshold based segmentation technique. The forth section covers the combination of SOM and the Hybrid Dynamic Genetic Algorithm. In the fifth section the experimental results are presented, and finally the conclusion.

\section{Image segmentation using Self-Organizing Maps (SOM)}

Kohonen's Self-Organizing Maps (SOM) (Kohenen, 2001) is an unsupervised neural network method. SOM converts patterns of arbitrary dimensionality into the responses of two dimensional arrays of neurons. One of the important characteristics of SOM is that the feature map preserves neighborhood relations of the input pattern. A typical SOM structure is given in Figure 1. It consists of two layers: an input layer and an output layer. The number of input neurons is equal to the dimensions of the input data. The output neurons are, however, arranged in a two-dimensional array.

Colors are one of the most important features considered in biological visual systems, since it is used to separate objects and patterns, even in conditions of equi-luminance (Levin, 1985). SOM is used to map patterns in a three-dimensional color (multi-bands) space to a two-dimensional space. In SOM, the input signals are n-tuples and there is a set of $m$ cluster units (automatic empirical determination with respect to the size of the satellite image). Each input is fully connected to all units. The initial weights are random and small, and their contribution for the final state decreases with the decrease of the number of samples (Yin \& Allinson, 1995). The network is composed of an orthogonal grid of cluster units (neurons), each is associated with three internal weights for the three layers of the satellite image. At each step in the training phase, the cluster unit with weights that best match the input pattern is elected as the winner usually by using minimum Euclidean distance as in Equation (1) 


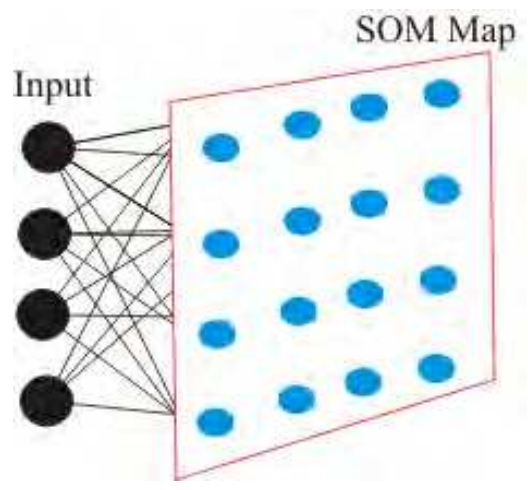

Fig. 1. Self-Organizing Map

$$
\left\|\mathbf{x}-W_{l}^{\lfloor k\rfloor}\right\|=\min _{i}\left\|\mathbf{x}-W_{i}^{\lfloor k\rfloor}\right\|
$$

Where $\mathbf{X}$ is the input vector, $W_{l}^{\lfloor k\rfloor}$ is the weight of the wining unit $l$ at iteration $k$, and $W_{i}^{\lfloor k\rfloor}$ is the weight for neuron $i$ at iteration $k$. The winning unit and a neighborhood around it are then updated in such a way that their internal weights be closer to the presented input. All the neurons within a certain neighborhood around the leader participate in the weightupdate process. This learning process can be described by the iterative procedure as in Equation (2).

$$
W_{i}^{\lfloor k+1\rfloor}=W_{i}^{\lfloor k\rfloor}+H_{l i}^{\lfloor k\rfloor}\left(\mathbf{x}-W_{i}^{\lfloor k\rfloor}\right)
$$

Where $H_{l i}^{\lfloor k}$ is a smoothing kernel defined over winning neuron. This kernel can be written in terms of the Gaussian function as in Equation 3.

$$
H_{l i}^{\lfloor k\rfloor}=\alpha^{\lfloor k\rfloor} \exp \left(-\frac{d^{2}(l, i)}{2\left(\sigma^{\lfloor k\rfloor}\right)^{2}}\right)
$$

$H_{l i}^{\lfloor k\rfloor} \rightarrow 0$ when $k \rightarrow T o$, where To is the total number of iterations. $\alpha^{\lfloor 0\rfloor}$ is the initial learning rate and it is equal to 0.1 . The learning rate is updated every iteration. $\sigma^{\lfloor k\rfloor}$ is the search distance at iteration $k$, initially can be half the length of the network or the maximum of either the width or length of the image divided by two. $d(l, i)$ is the distance between the leader neuron $l$ and its neighbor $i$. As learning proceeds, the size of the neighborhood should be diminished until it encompasses only a single unit.

After SOM neural network converges to a balance state, the original image is mapped from a high color space to a smaller color space. The number of colors in this space is equal to the number of neurons of SOM network. The final weights vectors in the map as the new sample space. This new data set is used for clustering, and allows determining a set of cluster centers. 


\section{SOM and the Threshold technique}

In order to eliminate small clusters (clusters with few pixels) and to reduce over segmentation problem the following technique (T-Cluster) is implemented. This technique consists of several steps as follow:

1-After obtaining cluster centers by SOM the process of clustering starts by calculating the distance between the values of the cluster centers representing the sum of the three bands.

2-Two clusters are combined if the distance between their centers is less than a predefined threshold $T$.

3- If step two is correct, the minimum number of pixels is considered in the combination procedure, where the cluster with smaller number of pixels is merged with the larger one. Figure 2 shows more details of the merging procedure and Equation 4 explains distance calculation.

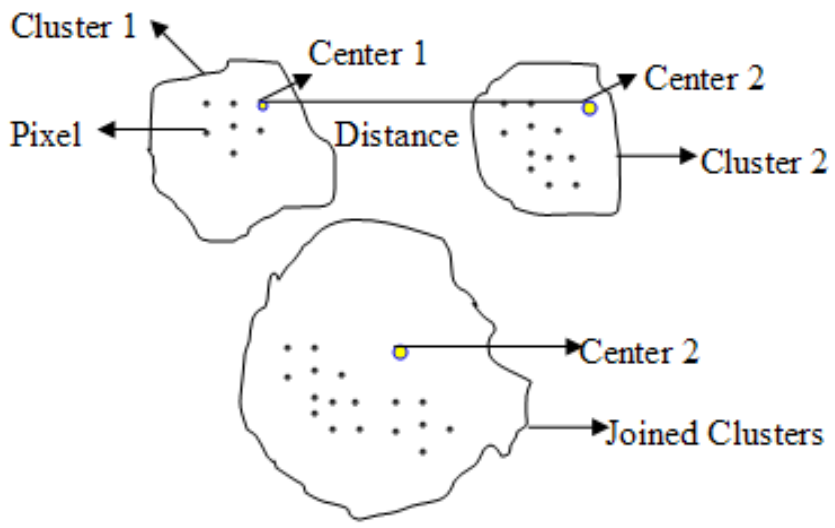

Fig. 2. Merging process according to the distance between cluster centers

$$
d\left(V\left(P_{i}\right), V\left(P_{j}\right)\right)<=T
$$

Where $T$ is a predefined threshold and $V\left(P_{i}\right)$ is the value of the three bands of the cluster center $P_{i}$. The value represents the sum of the resultant 3 weights obtained from running SOM each weight is multiplied by $255 . V\left(P_{j}\right)$ is the value of the three bands of another cluster center $P_{j}$. These two cluster centers are combined together if the distance value is less than a predefined threshold $T$. The value of the final cluster is the cluster with higher number of pixels.

SOM and the threshold technique (T-Cluster) work sequentially in order to complete the segmentation process. In other words, working separately cannot complete the job correctly (Figure 3). SOM uses satellite image features to organize pixels in group. The highest peaks of the histogram are used as cluster centers and are provided to T-Cluster to deliver the final solution in the image segmentation process.

This method starts by reading a satellite image than it is provided to SOM to organize pixels in groups. The organized pixels are used by T-Cluster to obtain the final number of cluster centers (no under or over segmentation). TSOM fixes the problem of under and over segmentation which are caused by using SOM separately. 


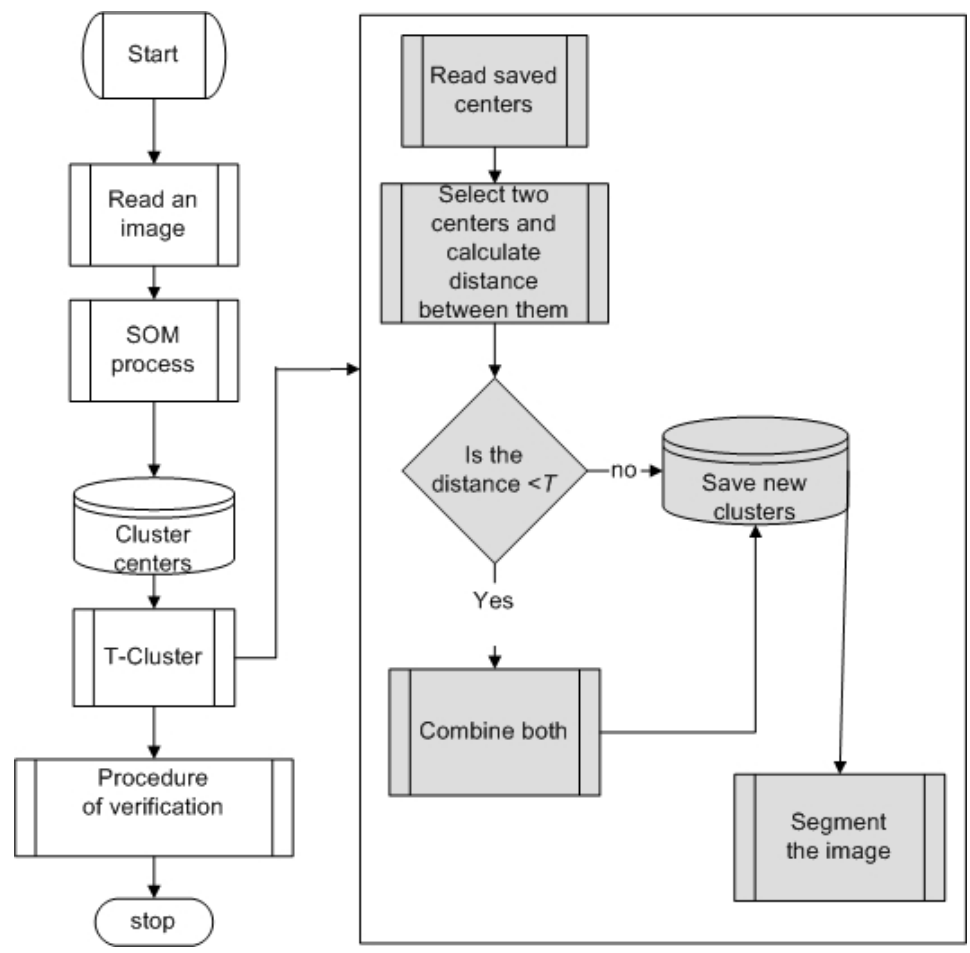

Fig. 3. SOM and T-Cluster sequential process

\section{SOM and Hybrid Dynamic Genetic Algorithm}

Accuracy obtained using only SOM in image segmentation may often be unsatisfactory (Awad et al., 2009b). So, in order to improve the result of satellite image segmentation, SOM and HDGA (Awad et al., 2009a) work sequentially in order to achieve the highest accuracy (See Figure 4 for complete details).

First, the process starts by reading a satellite image, and then SOM uses multi-component features of the image to organize the image pixels in groups. Each group value is used as a cluster center and is provided to the Hybrid Dynamic Genetic Algorithm (HDGA) for selecting the optimal solution to select the optimal image segmentation solution. HDGA is a modified Genetic Algorithm (GA) to become Hybrid by adding Hill-climbing process. Moreover, a heuristic process which increases the number of feasible solutions is added in order to speed up the process of escaping local optima solution. The chromosomes which form the population of the Hybrid Dynamic Genetic Algorithm (HDGA) consist of different number of cluster centers, which means that many different solutions are available. In the previous method the success of the segmentation process depends on the correct selection of two criteria:

1- The minimum number of pixels in each group and 2- The degree of similarity of the grey level values of the cluster centers. 
These two criteria were necessary and they had to be adjusted in the previous method using the threshold technique. But, this concern is eliminated by using HDGA.

HDGA creates population of chromosomes where each four genes represent the cluster center provided by SOM and the other three genes represent the grey level value for each pixel in the three bands in the satellite image. In each iteration, the chromosomes are evaluated using (5) and the best solution is selected.

$$
O F=\operatorname{Min}\left(\sum_{j=1}^{k} \sum_{i=0}^{n}\left[\sum_{y=1}^{3} V\left(P_{j y}\right)-\sum_{z=1}^{3} V\left(p x_{i z}\right)\right]\right)
$$

Where $k$ is the number of the centers in a chromosome and $V\left(P_{j y}\right)$ is the value of the cluster center $P_{j}$ in each band of the 3 bands of the image. $V\left(p x_{i z}\right)$ is the grey level values of the pixel in each band of the 3 bands of the image to the left of the cluster center $P_{j}$ in the chromosome. Variable $n$ is the number of pixels in each cluster $\quad\left(\ldots p x_{i 1} p x_{i 2} p x_{i 3} P_{j 1} P_{j 2} P_{j 3}\right.$ $\left.p x_{(i+1) 1} p x_{(i+1) 2} p x_{(i+1) 3} P_{j 1} P_{j 2} P_{j 3} \ldots\right)$.

SOM-HDGA fixes the problem of under and over segmentation caused by using one method alone as will be proved later in the experiments.

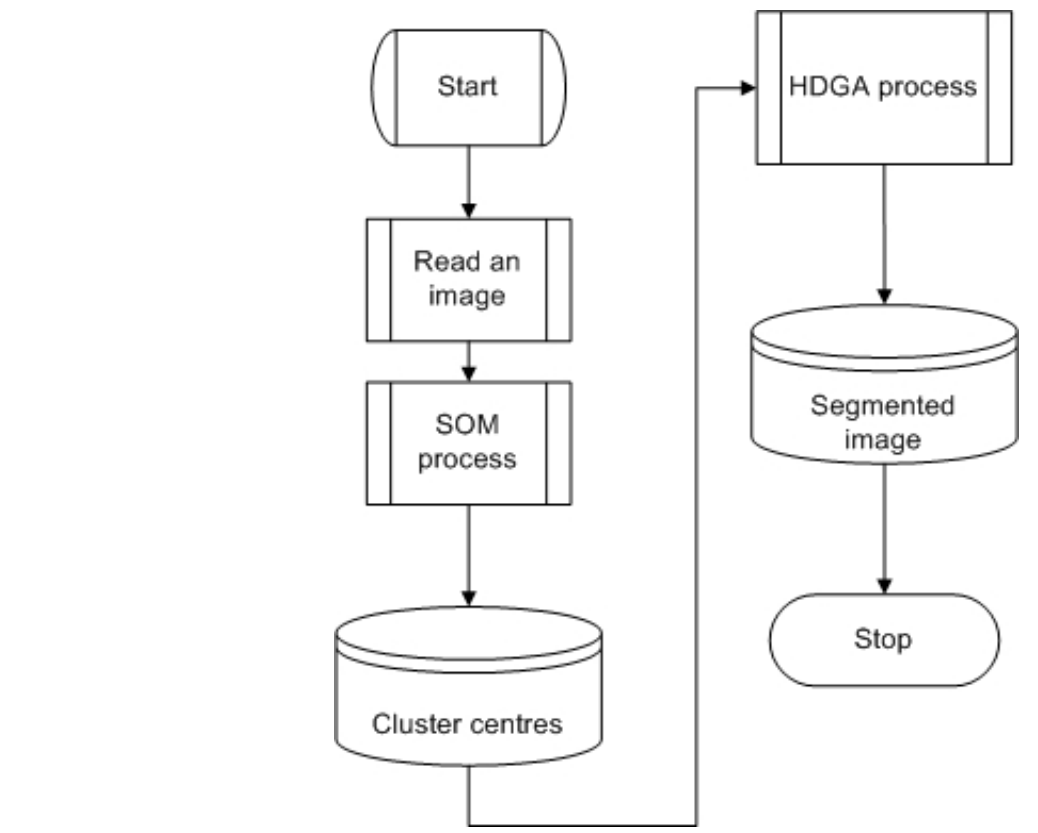

Fig. 4. SOM and HDGA cooperative method

\section{Experimental results}

The proposed image segmentation methods are implemented using $\mathrm{C}$ language on an Intel Centrino $^{\mathrm{TM}}-1.7 \mathrm{GHz}$ computer. The number of iterations for SOM is 1000, and HDGA will be terminated when the fitness value of the best individual remains unchanged over the 
past 20 generations. Three experiments are conducted using two types of satellite images (Spot-5 and Ikonos-II). Spot-5 started orbiting earth in 2002. The image captured by Spot-5 consists of 3 bands, of $5 \mathrm{~m}$ resolution pan-sharpened by a panchromatic band of $2.5 \mathrm{~m}$ resolution). Spot-5 is commonly referred to as a pushbroom scanner meaning that all scanning parts are fixed, and scanning is accomplished by the forward motion of the scanner. Ikonos-II is an important high-resolution satellite operated by GeoEye formerly Space Imaging LLC. Its capabilities include capturing a $3.2 \mathrm{~m}$ multispectral, near-infrared (NIR) and $1 \mathrm{~m}$ panchromatic resolution.

The experiments are conducted in order to demonstrate the accuracy, and efficiency of SOM with the clustering technique and cooperating with another method. The results are compared to that of the Iterative Self-organizing Data (ISODATA) algorithm. ISODATA clustering is iterative in that it repeatedly performs an entire classification (outputting a thematic raster layer) and recalculates statistics. Self-Organizing refers to the way in which it locates clusters with minimum user input. The ISODATA method uses minimum spectral distance to assign a cluster for each candidate pixel. The process begins with a specified number of arbitrary cluster means or the means of existing signatures, and then it processes repetitively, so that those means shift to the means of the clusters in the data. The ISODATA algorithm has some further refinements by splitting and merging of clusters (Jensen, 1996). At the end of the segmentation processes, several samples are collected from the segmented images representing different major classes. These samples are verified using a Global Positioning System (GPS) device with high accuracy (2 meter) and confusion matrices (Kohavi \& Provost, 1998) which contain information about actual and predicted segmentation done by a segmentation method. The actual values are represented in the columns and the predicted values are represented in the rows of the matrix. Performance of such systems is commonly evaluated using the data in the matrix. Samples are selected from the segmented image based on the size of the area and the ambiguity of the results. The bigger the area or the more ambigeous the more samples are collected, and the smaller or the clearer the area the less samples are collected.

It is well known that when the ratio of the number of training samples to the number of feature measurements is small, the estimates of the discriminant functions are not accurate this is called Hughes phenomenon (Shahshahani \& Landgrebe, 1994).

\subsection{Spot $V$ image segmentation}

The first test image is a Spot $V$ image with size of $360 \times 360$ pixels (see Figure $5(a)$ ). The image represents an area near an airport. ISODATA segmentation result is shown in Figure 5(b), TSOM segmentation result is shown in Figure 5(c), and SOM-HDGA segmentation result is shown in Figure 5(d). In order to compare these experimental results of ISODATA, TSOM, and SOM-HDGA segmentation methods, a confusion matrix is used where terrain verification is applied and compared with the results of these segmentation methods. This process is accomplished using four different classes ( $1=$ Vegetation type I (dark green), $2=$ Vegetation type II (light green), 3= Soil (brown color), 4= Urban (tan)) and 400 survey points (see Tables 1, 2, and 3 respectively). The accuracies of the three different segmentation methods are $78 \%, 84 \%$, and $90 \%$ respectively. 


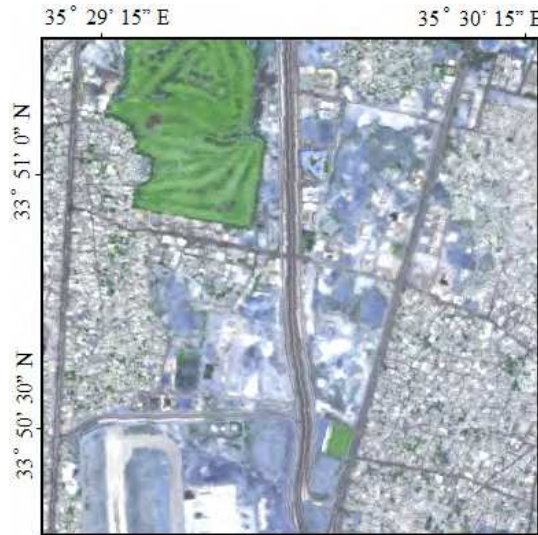

(a) Original image

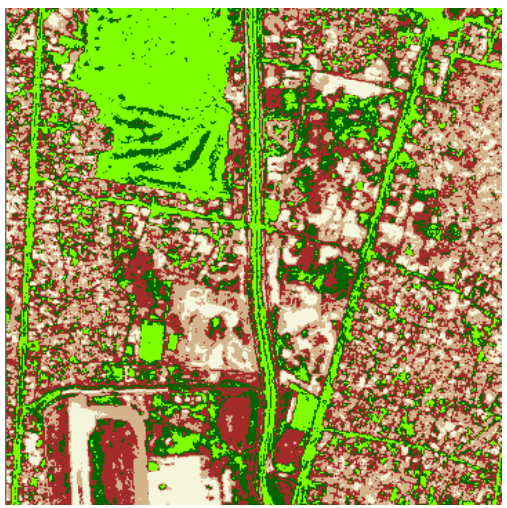

(c) TSOM result

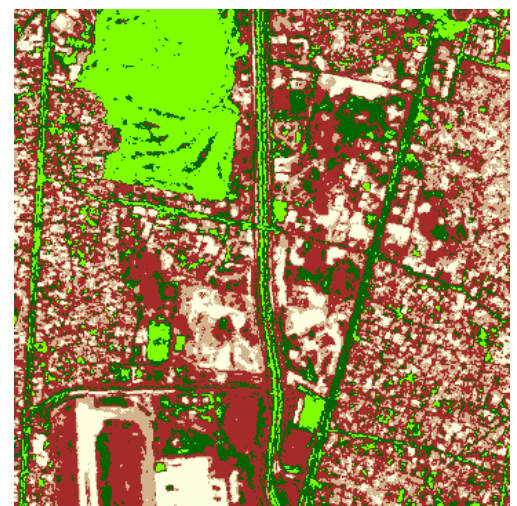

(b) ISODATA result

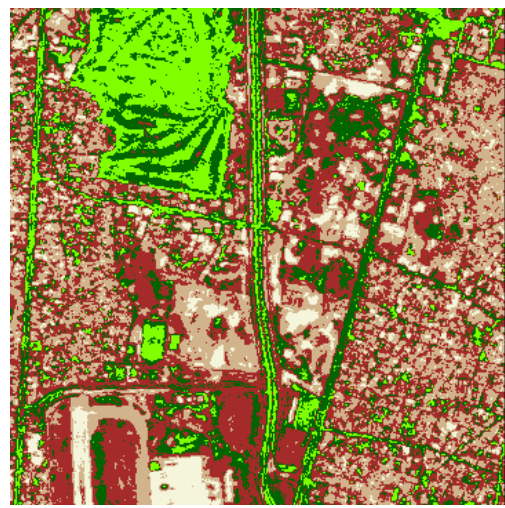

(d) SOM-HDGA result

Fig. 5. Spot V satellite image and the results of the different segmentation methods

\begin{tabular}{|l|l|l|l|l|l|}
\hline Ground truth classes & 1 & 2 & 3 & 4 & Total \\
\hline 1- Vegetation- type 1 & 80 & 10 & 2 & 8 & 100 \\
\hline 2- Vegetation- type 1I & 5 & 78 & 5 & 12 & 100 \\
\hline 3-Soil & 7 & 7 & 80 & 6 & 100 \\
\hline 4- Urban & 12 & 9 & 4 & 75 & 100 \\
\hline Total & 104 & 104 & 91 & 101 & 400 \\
\hline
\end{tabular}

Table 1. Confusion matrix for ISODATA

\begin{tabular}{|l|l|l|l|l|l|}
\hline Ground truth classes & 1 & 2 & 3 & 4 & Total \\
\hline 1- Vegetation- type 1 & 84 & 7 & 2 & 7 & 100 \\
\hline 2- Vegetation- type 1I & 2 & 83 & 5 & 10 & 100 \\
\hline 3-Soil & 5 & 6 & 83 & 6 & 100 \\
\hline 4- Urban & 6 & 4 & 5 & 85 & 100 \\
\hline Total & 97 & 100 & 95 & 108 & 400 \\
\hline
\end{tabular}

Table 2. Confusion matrix for TSOM 


\begin{tabular}{|l|l|l|l|l|l|}
\hline Ground truth classes & 1 & 2 & 3 & 4 & Total \\
\hline 1- Vegetation- type 1 & 86 & 4 & 4 & 6 & 100 \\
\hline 2- Vegetation- type 1I & 1 & 91 & 2 & 6 & 100 \\
\hline 3-Soil & 1 & 3 & 94 & 2 & 100 \\
\hline 4- Urban & 3 & 4 & 5 & 88 & 100 \\
\hline Total & 91 & 102 & 105 & 102 & 400 \\
\hline
\end{tabular}

Table 3. Confusion matrix for SOM-HDGA

\subsection{Ikonos-II image segmentation}

The second test image is an Ikonos-II image with size $360 \times 360$ pixels. This image represents a coastal area in the Mediterranean Basin. The image is selected because of the diversity of classes (urban of different types and structures, vegetation, sand, soil...etc) which creates a complex texture. In addition, the goal is to minimize the effects of the existing of shadows which are the main problem in high resolution images. The image consists of the visible bands pan-sharpened with the panchromatic band (see Figure 6(a)). ISODATA segmentation result is shown in Figure 6(b), TSOM segmentation result is shown in Figure 6(c), and SOM-HDGA segmentation result is shown in Figure 6(d).

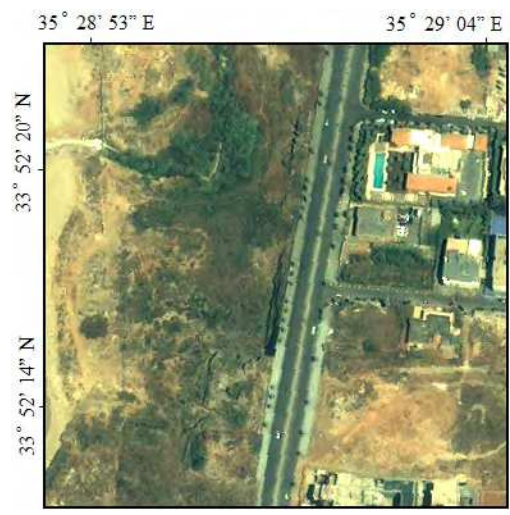

(a) Original image

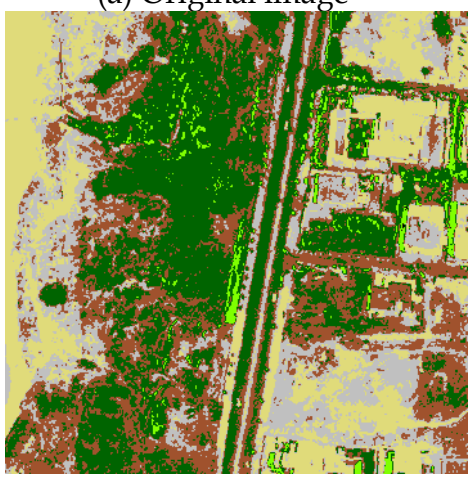

(c) TSOM result

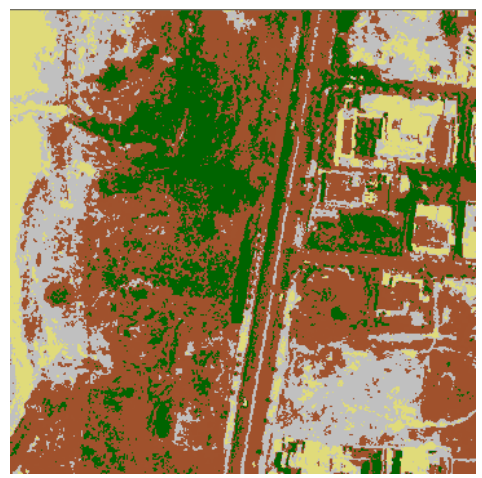

(b) ISODATA result

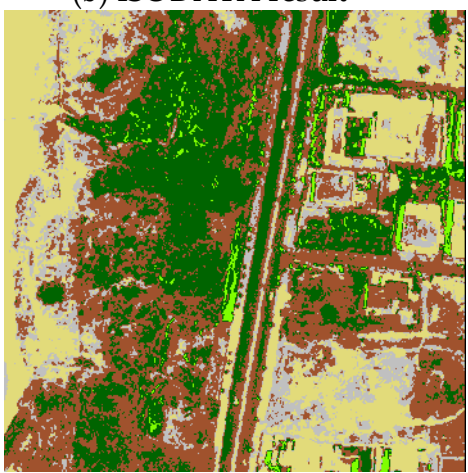

(d) SOM-HDGA result

Fig. 6. Ikonos-II satellite image and the results of different segmentation methods 
One can notice easily that shadow is mixed with vegetation in ISODATA while it is well separated in the other two segmentation methods (light green). In addition, ISODATA is not separating vegetation properly as compared to the other two methods. Again, the same procedure is used to verify the results of the three segmentation methods. Four classes are selected (1- high to medium dense vegetation "dark green", 2- Sparse vegetation "brown", 3- Sand type I and urban "gray", 4- Sand type II and urban "light yellow"). The total number of samples for the 4 classes is 320 distributed evenly between the classes. However, some areas are surveyed extensively in order to clarify the ambiguity which are found in the results (i.e. the segmentation of sandy and shrubs areas are clearly different between the three methods).

The confusion matrices for the results of the three segmentation methods are shown in table 4,5 , and 6 respectively.

\begin{tabular}{|l|l|l|l|l|l|}
\hline Ground truth classes & 1 & 2 & 3 & 4 & Total \\
\hline 1- Vegetation- type 1 & 51 & 20 & 5 & 4 & 80 \\
\hline 2- Vegetation- type 1I & 22 & 49 & 7 & 2 & 80 \\
\hline 3- Sand type I/urban & 2 & 3 & 60 & 15 & 80 \\
\hline 4- Sand type II/urban & 10 & 5 & 13 & 52 & 80 \\
\hline Total & 85 & 77 & 85 & 73 & 320 \\
\hline
\end{tabular}

Table 4. Confusion matrix for ISODATA

\begin{tabular}{|l|l|l|l|l|l|}
\hline Ground truth classes & 1 & 2 & 3 & 4 & Total \\
\hline 1- Vegetation- type 1 & 69 & 7 & 3 & 1 & 80 \\
\hline 2- Vegetation- type 1I & 8 & 65 & 4 & 3 & 80 \\
\hline 3- Sand type I/urban & 1 & 4 & 68 & 7 & 80 \\
\hline 4- Sand type II/urban & 1 & 2 & 5 & 72 & 80 \\
\hline Total & 79 & 78 & 80 & 83 & 320 \\
\hline
\end{tabular}

Table 5. Confusion matrix for TSOM

\begin{tabular}{|l|l|l|l|l|l|}
\hline Ground truth classes & 1 & 2 & 3 & 4 & Total \\
\hline 1- Vegetation- type 1 & 72 & 4 & 3 & 1 & 80 \\
\hline 2- Vegetation- type 1I & 5 & 70 & 3 & 2 & 80 \\
\hline 3- Sand type I/urban & 1 & 3 & 71 & 5 & 80 \\
\hline 4- Sand type II/urban & 1 & 1 & 3 & 75 & 80 \\
\hline Total & 79 & 78 & 80 & 83 & 320 \\
\hline
\end{tabular}

Table 6. Confusion matrix for SOM-HDGA

The accuracies of the three segmentation methods are computed from the matrices and they are $66 \%$ for ISODATA, $86 \%$ for TSOM, and 90\% for SOM-HDGA.

\section{Conclusion and future work}

Segmentation is an important step in image processing. The lack of an efficient unsupervised non-parametric method to segment any type of satellite images specifically 
high resolution images led us to implement two different sequential methods for satellite image segmentation. These methods are TSOM and SOM-HDGA. When applying these methods to satellite images, the results are more robust and efficient than those obtained with ISODATA segmentaton/classification method. SOM-HDGA overall average efficiency is equal or greater than $90 \%$. However the efficiency of ISODATA degrades with the increase of image resolution. The overall average of ISODATA is about $66 \%$. SOM-HDGA process robustness and efficiency are due to the fact that a primary solution is provided by SOM to HDGA which in turn uses only the best organized data by avoiding the use of small clusters.

On the other hand, in ISODATA an initial number of clusters must be given for the technique to work. SOM-HDGA performance can be improved using parallel cooperation with more segmentation methods such as Fuzy C-Means. Another approach to improve the segmentation process is to use a knowledge base which contains the cluster centers of previous segmentation on different satellite images with similar date. This is very important for multi-resolution satellite image segmentation. In addition, the knoweledge base may have information about not only the capturing date of the image, but other related information, which will help in speeding up the process of segmenting similar satellite images. This information may be about fixed and variable classes e.g. Forests for fixed classes and arable land for variable classes.

\section{References}

Aria, E.; Saradjian, M., Amini, J. \& Lucas, C. (2004). Generalized Cooccurence matrix to classify IRS-1D images using Neural Network, Proceedings of XXth ISPRS Congress, pp. 117-123, Turkey.

Awad, M.; Chehdi, K. \& Nasri, A. (2007). Multi-component image segmentation using Genetic Algorithm and Artificial Neural Network. IEEE Geosciences and Remote Sensing Letters, Vol. 4, no. 4, pp. 571-575, 2007.

Awad, M.; Chehdi, K. \& Nasri, A. (2009a). Multi-component Image Segmentation Using a Hybrid Dynamic Genetic Algorithm and Fuzzy C-Means. IET image processing, Vol. 3, No. 2, pp. 52-62.

Awad, M.; Chehdi, K. \& Nasri, A. (2009b). Satellite Image Segmentation: a Comparative Analysis between SOM \& HGA. International Journal of Remote Sensing, Vol. 30, No. 3, pp. 595-610.

Benediktsson, J.; Swain, P., Ersoy, O. \& Hong, D. (1990). Neural Network Approaches Versus Statistical Methods in Classification of Multisource Remote Sensing Data. IEEE Transactions on Geoscience and Remote Sensing, Vol. 28, No. 4, pp. 540-551.

Jensen, J. (1996). Introductory Digital Image Processing: A Remote Sensing Perspective. Englewood Cliffs, New Jersey: Prentice-Hall.

Kohavi, R. \& Provost, F. (1998). Glossary of Terms. Special Issue on Applications of Machine Learning and the Knowledge Discovery Process, Vol. 30, No. 2/3.

Kohenen, T. (2001). Self-Organizing Maps. Springer Series Information Sciences, Vol. 30, 501 pages.

Levine, M. (1985). Vision in man and machine, McGraw Hill Publ. Co., New York. 
Lopes, A.; Nezry, E., Touzi, R. \& Laur, H. (1990). Maximum A Posteriori Speckle Filtering and First Order Textural Models in SAR Images, Poceedings of International Geoscience and Remote Sensing Symposium (IGARSS), vol. 3, pp. 2409-2412, Maryland, USA.

Mason, P. and Pieczynski, W. (1993). SEM algorithm and unsupervised segmentation of satellite images. IEEE Transactions on Geoscience and Remote Sensing, Vol. 31, pp. 618633.

Perkins, S.; Theiler, J., Brumby, S., Harvey, N., Porter, R., Szymansk, J. \& Bloch, J. (2000). GENIE: A Hybrid Genetic Algorithm for Feature Classification in Multi-Spectral Images, Proceedings of SPIE Applications and Science of Neural Networks, Fuzzy Systems, and Evolutionary Computation III 4120, pp 52-62, USA.

Pratt, W. (1991). Digital Image Processing 2d ed, John Wiley \& Sons, Inc., New York.

Shahshahani, M. \& Landgrebe, A. (1994). The effect of unlabeled samples in reducing the small sample size problem and mitigating the Hughes phenomenon. IEEE Transactions on Geoscience and Remote Sensing, Vol. 32, pp. 1087- 1095.

Tou, J. \& Gonzalez, R. (1974). Pattern Recognition Principles, Massachusetts: Addison-Wesley Publishing Company.

Xu, B. \& Lin, S. (2002). Automatic color identification in printed fabric images by a fuzzyneural network. AATICC Review, Vol. 2, No. 9, pp. 42-45.

Yao, K.; Mignotte, M., Collet, C., Galerne, P. \& Burel, G. (2000). Unsupervised segmentation using a self-organizing map and a noise model estimation in sonar imagery. Pattern Recognition Letters, Vol. 33, No. 9, pp. 1575-1584.

Yin, H. \& Allinson, N. (1995). On the distribution and convergence of feature space in selforganizing maps. Neural Computation, Vol. 7, No. 6, pp. 1178-1187.

Zhang, P.; Verma, B. \& Kumar, K. (2003). Neural vs Statistical Classifier in Conjunction with Genetic Algorithm Feature Selection in Digital Mammography, Proceedings of IEEE Congress on Evolutionary Computation, CEC'03, pp. 634- 639, Australia.

Zhou, Z.; Wei, S., Zhang, X. and Zhao, X. (2007). Remote sensing image segmentation based on self-organizing map at multiple-scale, Proceedings of SPIE Geoinformatics: Remotely Sensed Data and Information 6752, USA. 


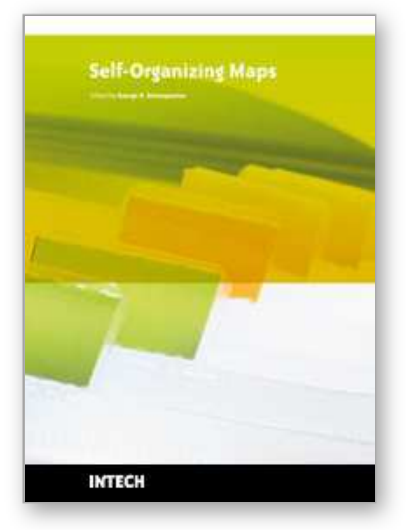

\author{
Self-Organizing Maps \\ Edited by George K Matsopoulos
}

ISBN 978-953-307-074-2

Hard cover, 430 pages

Publisher InTech

Published online 01, April, 2010

Published in print edition April, 2010

The Self-Organizing Map (SOM) is a neural network algorithm, which uses a competitive learning technique to train itself in an unsupervised manner. SOMs are different from other artificial neural networks in the sense that they use a neighborhood function to preserve the topological properties of the input space and they have been used to create an ordered representation of multi-dimensional data which simplifies complexity and reveals meaningful relationships. Prof. T. Kohonen in the early 1980s first established the relevant theory and explored possible applications of SOMs. Since then, a number of theoretical and practical applications of SOMs have been reported including clustering, prediction, data representation, classification, visualization, etc. This book was prompted by the desire to bring together some of the more recent theoretical and practical developments on SOMs and to provide the background for future developments in promising directions. The book comprises of 25 Chapters which can be categorized into three broad areas: methodology, visualization and practical applications.

\title{
How to reference
}

In order to correctly reference this scholarly work, feel free to copy and paste the following:

Mohamad Awad (2010). Segmentation of Satellite Images Using Self-Organizing Maps, Self-Organizing Maps, George K Matsopoulos (Ed.), ISBN: 978-953-307-074-2, InTech, Available from:

http://www.intechopen.com/books/self-organizing-maps/segmentation-of-satellite-images-using-self-

organizing-maps

\section{INTECH}

open science | open minds

\section{InTech Europe}

University Campus STeP Ri

Slavka Krautzeka 83/A

51000 Rijeka, Croatia

Phone: +385 (51) 770447

Fax: +385 (51) 686166

www.intechopen.com

\section{InTech China}

Unit 405, Office Block, Hotel Equatorial Shanghai

No.65, Yan An Road (West), Shanghai, 200040, China

中国上海市延安西路65号上海国际贵都大饭店办公楼405单元

Phone: +86-21-62489820

Fax: $+86-21-62489821$ 
(C) 2010 The Author(s). Licensee IntechOpen. This chapter is distributed under the terms of the Creative Commons Attribution-NonCommercialShareAlike-3.0 License, which permits use, distribution and reproduction for non-commercial purposes, provided the original is properly cited and derivative works building on this content are distributed under the same license. 